Pacific Journal of Mathematics

COMPLEX VECTOR FIELDS AND DIVISIBLE CHERI 


\title{
COMPLEX VECTOR FIELDS AND DIVISIBLE CHERN CLASSES
}

\author{
Robert D. LitTle
}

This paper contains two theorems which relate the maximal number of independent sections of a complex bundle over a manifold to the Chern classes of the bundle and certain functional cohomology operations. The main theoretical result of the paper is a formula which relates the obstruction to a lifting in a fibration and a functional cohomology operation.

1. Introduction. Let $M$ be a connected, closed, orientable, smooth manifold of dimension $2 n$. If $\omega$ is a complex $n$-plane bundle over $M$, the complex span of $\omega$ is the maximal number of cross-sections of $\omega$ which are linearly independent over the complex numbers. In this paper, we consider the following question: when is complex span $\omega \geqq q$ ? Hopf's theorem says that complex span $\omega>0$ if and only if $\omega$ has vanishing Euler class and the theorems of Thomas ([10] and [11]) give an effective answer in the case $q=2$. We study this problem in the cases $q=3,4$ and establish theorems which give necessary and sufficient conditions for complex span $\omega \geqq q$ in terms of the Chern classes of $\omega$ and certain functional cohomology operations. The Chern class of $\omega$ in $H^{2 i}(M ; \mathbf{Z})$ is denoted by $c_{l}(\omega)$. If $\delta_{p} P^{1}$ denotes the Steenrod $p$-power $P^{1}$ followed by the Bockstein associated with reduction $\bmod p, \delta_{p} P_{\omega}^{1}(c(i-$ $p+1)$ ) denotes a subset of a functional operation defined on the universal Chern class $c(i-p+1)$ and contained in $H^{2 i}(M ; Z)$. This subset will be described in the second section of this paper. If $p$ is a prime, $M$ is $j$-connected $\bmod p$ if $H_{t}\left(M ; \mathbf{Z}_{p}\right)=0,1 \leqq i \leqq j . \quad$ In both theorems below, $M$ is 1 -connected mod 2 and 3.

THEOREM 1. If $n$ is even, $n \geqq 6$, then complex span $\omega \geqq 3$ if and only if $c_{\iota}(\omega)=0, n-2 \leqq i \leqq n$, and $0 \in \delta S q_{\omega}^{2}(c(n-2))$.

THEOREM 2. If $n$ is odd, $n \neq 1(\bmod 3), n \geqq 9$, and $M$ is 3 -connected $\bmod 2$, then complex span $\omega \geqq 4$ if and only if $c_{t}(\omega)=0, n-3 \leqq i \leqq n$, $0 \in \delta S q_{\omega}^{2}(c(n-3))$, and $0 \in \delta_{3} P_{\omega}^{1}(c(n-3))$.

In Theorem 1 , if $n \neq 0(\bmod 3)$, and $n \equiv 2(\bmod 4)$, the connectedness hypothesis can be dropped. Thomas and Gilmore [11] show that if $M$ is 3 -connected, then for every $n$, complex $\operatorname{span} \omega \geqq 3$ if and only if $c_{n-2}(\omega)=0$ and $c_{n}(\omega)=0$. Gilmore [2] proves theorems similar to Theorems 1 and 2 in which he assumes that $H_{2}(M ; \mathbf{Z})$ and $H_{4}(M ; \mathbf{Z})$, 
respectively, have no elements of order 2. For the specified values of $n$, our theorems contain the results of Thomas and Gilmore, because the operation $\delta_{p} P_{\omega}^{1}(c(i-p+1))$ is a set of elements of order $p$ and hence must vanish if $H^{2 t}(M ; \mathbf{Z})$ has no $p$-torsion. If $M$ is an almost-complex manifold with almost-complex structure $\omega$, our theorems relate the complex span of $M$ to the Chern classes of $M$ and the functional operations.

2. Obstruction formulas. Let the map $f: M \rightarrow B U(n)$ classify $\omega$. It is clear that complex span $\omega \geqq q$ if and only if $f$ lifts to the total space of the fibration $W_{n, q} \rightarrow B U(n-q) \rightarrow B U(n)$ where $W_{n, q}$ is the Stiefel variety of complex $q$-frames in complex $n$-space. We will apply two obstruction formulas to this lifting problem. The first formula is due to Olum [8]. If $\pi: E \rightarrow B$ is a fibration with fiber $F, c$ in $H^{n-1}(F ; G)$ a class transgressing to $d$ in $H^{n}(B ; G), X$ a $C W$ complex and $f: X \rightarrow B$ a map such that the lifting obstruction $O^{n}(f)$ is nonvoid, then

$$
-c_{*} O^{n}(f)=f^{*} d,
$$

where $c_{*}: H^{n}\left(X ; \pi_{n-1}(F)\right) \rightarrow H^{n}(X ; G)$ is induced by $c_{\#}: \pi_{n-1}(F) \rightarrow G$, the composite of the Hurewicz homomorphism and evaluation.

The second formula is the main theoretical result of this paper. We assume that there is a class $a$ in $H^{n-2 p+1}(F ; \mathbf{Z})$ such that $\delta P^{1} a=0$ and $a$ is the only class transgressing to $b$, where $f^{*} b=0$ and $P^{1} b \equiv 0(\bmod$ integral classes in kernel $\pi^{*} \cap$ kernel $f^{*}$ ). Under these hypotheses, there are liftings $f_{a}: F \rightarrow K\left(\mathbf{Z}, n-2 p+1 ; \mathbf{Z}, n-1, \delta P^{1}\right)$ and $f_{b}: B$ $\rightarrow K\left(\mathbf{Z}, n-2 p+2 ; \mathbf{Z}, n, \delta P^{1}\right)$ of $a$ and $b$, where the range spaces are two-stage Postnikov systems induced by $\delta P^{1}$. In [3], we show that the set $\left\{f_{a \neq t}: \pi_{n-1}(F) \rightarrow \mathbf{Z}\right\}$ is a congruence class modulo the image of the Hurewicz homomorphism and for every $[g]$ in $\pi_{n-1}(F), f_{a *}[g] \in \delta P_{g}^{1}(a)$, where $\delta P_{g}^{1}$ is the standard functional operation. (See [6], p. 157.) Therefore the induced homomorphism $f_{a^{*}}: H^{n}\left(X ; \pi_{n-1}(F)\right) \rightarrow H^{n}(X ; \mathbf{Z})$ can be effectively computed in some cases. In the proposition below, $\iota$ denotes the fundamental class of $K\left(\mathbf{Z}, n-2 p+2 ; \mathbf{Z}, n, \delta P^{1}\right)$ and we assume that $H^{n}(E ; \mathbf{Z})$ and $H^{n}(B ; \mathbf{Z})$ are torsion free.

Proposition 2.2. If $\pi: E \rightarrow B$ is a fibration satisfying the above hypotheses and $p$ is a prime such that $n \geqq 2(2 p-1)$, then we have containments

$$
f_{a^{*}} O^{n}(f) \equiv \delta P_{f_{b}}^{1}(\iota) \equiv \delta P_{f}^{1}(b) .
$$

Note that (2.3) shows that the obstruction is contained in the 
operation $\delta P_{f}^{1}(b)$. A general result of this kind was obtained by Meyer. (See [5], §13.) It will be clear in the proof of (2.2) that the indeterminacy of $\delta P_{f_{b}}^{1}$ is image $\delta P^{1}$, and so (2.3) expesses the obstruction as an operation with smaller indeterminacy than the indeterminacy of $\delta P_{f}^{1}$, image $\left(\delta P^{1}+f^{*}\right)$. Proposition 2.2 will follow from the next lemma. In the proof of the lemma, the reduction $\bmod p$ of an integral class $\theta$ will be denoted by $\bar{\theta}$.

\section{LEMMA 2.4. The composite $f_{b} \pi$ is homotopically trivial.}

Proof. Let $\quad K=K\left(\mathbf{Z}, n-2 p+2 ; \mathbf{Z}, n, \delta P^{1}\right) . \quad$ If $\quad n \geqq 2(2 p-1)$, $H^{n}(K ; \mathbf{Z})$ is isomorphic to $\mathbf{Z}$ modulo finite groups with a free generator $\theta$ such that $\theta_{\sharp}: \pi_{n}(K) \rightarrow \mathbf{Z}$ is multiplication by $p$ and $\bar{\theta}=P^{1} \iota$. These facts follow immediately from the long exact homotopy and Serre cohomology sequences for the fibration $K \rightarrow K(\mathbf{Z}, n-2 p+2)$ and the Hurewicz theorem modulo finite groups. Since $\left(f_{b} \pi\right)^{*} \iota=\pi^{*} b=0$, because $b$ is in the image of the transgression, $O^{n}\left(f_{b} \pi, *\right)$ is nonvoid. It follows from the hypotheses that $f_{b}^{*} \bar{\theta} \equiv 0\left(\bmod\right.$ integral classes in kernel $\pi^{*} \cap$ kernel $\left.f^{*}\right)$ and from the properties of $\theta$ and (4.4) in [8] that $f_{b}^{*} \theta-f_{b}^{\prime *} \theta=$ $p O^{n}\left(f_{b}, f_{b}^{\prime}\right)$ for any two liftings of $b$. Therefore, after alteration by an $n$-cocycle, we may assume $f_{b}^{*} \theta \in$ kernel $\pi^{*} \cap$ kernel $f^{*}$ and so $\pi^{*} f_{b}^{*} \theta=$ $0=p O^{n}\left(f_{b} \pi, *\right)$, and this implies $f_{b} \pi$ is homotopically trivial since $H^{n}(E ; \mathbf{Z})$ has no torsion.

Lemma 2.4 implies the existence of a map of fibrations from the fibration $\pi$ into the path space fibration over $K$ with fiber $\Omega K=$ $K\left(\mathbf{Z}, n-2 p+1 ; \mathbf{Z}, n-1, \delta P^{1}\right)$. The map of fibers is a lifting of $a$, $f_{a}: F \rightarrow K\left(\mathbf{Z}, n-2 p+1 ; \mathbf{Z}, n-1, \delta P^{1}\right)$, because we are assuming that $a$ is the only class transgressing to $b$. The map $f_{b} f$ lifts to the path space if and only if it is homotopic to the constant map. We have taken care that $f_{b}^{*} \theta \in \operatorname{ker} f^{*}$ and so $\left(f_{b} f\right)^{*}$ has image zero in dimension $n$. Therefore the obstruction to a homotopy of $f_{b} f$ to point is precisely $\delta P_{f_{b} f}^{1}(\iota)(10.8$ [7]) and the indeterminacy of $\delta P_{f_{b} f}^{1}$ is image $\delta P^{1}$. Formula (2.3) now follows immediately from standard naturality properties of obstructions and functional operations ([6], p. 157).

3. The proofs of Theorems 1 and 2. We begin with some general remarks. The group $H^{*}\left(W_{n, q} ; \mathbf{Z}\right)$ is an exterior algebra with generators $a_{k}$ in $H^{2 k-1}\left(W_{n, q} ; \mathbf{Z}\right), n-q+1 \leqq k \leqq n$ ([1], p. 444). The space $W_{n, q}$ is $2(n-q)$-connected and $\pi_{i}\left(W_{n, q}\right)$ is $\mathbf{Z}$ if $i$ is odd and a finite group if $i$ is even as long as $n$ is large, $n-q$ is odd, $2 \leqq q \leqq 4$, and $2(n-q)+1 \leqq i \leqq 2 n-1$. The necessary size of $n$ is indicated in the theorems. The group $\pi_{2(n-q)+2}\left(W_{n, q}\right)$ is zero and the other finite groups are 2 or 3 torsion groups in this range of dimensions, [2]. Since the 
Hurewicz homomorphism $h: \pi_{i}\left(W_{n, q}\right) \rightarrow H_{l}\left(W_{n, q} ; \mathbf{Z}\right)$ is a isomorphism modulo finite abelian groups if $i \leqq 4(n-q)$, it sends a generator in $\pi_{2 k-1}\left(W_{n, q}\right)$ into an integer $m_{k} \neq 0, n-q+1 \leqq k \leqq n$. These integers can be computed using an inductive argument based on the fibration $W_{n-1, q-1} \rightarrow W_{n, q} \rightarrow S^{2 n-1}$. In particular, $m_{n-q+1}=1, m_{n-q+2}=2$ and the prime divisors of the others are either 2 or 3, [4]. Since $a_{k}$ transgresses to $c(k)$, it follows from (2.1) that $-m_{k} O^{2 k}(f)=c_{k}(\omega)$. Thomas' theorem [10] follows from this formula and the first two values of $m_{k}$ : if $M$ is arbitrary and $n$ is odd, complex span $\omega \geqq 2$ if and only if $c_{i}(\omega)=0$, $n-1 \leqq i \leqq n$.

Consider the lifting obstruction $O^{2 k}(f)$, where $n-q+p \leqq k \leqq$ $n$. In this range of dimensions, $c(k-p+1)$ is the image of a unique class $a_{k-p+1}$ under transgression and if $c_{k}(\omega)=0, P^{1} c(k-p+1) \equiv 0(\bmod$ integral classes in kernel $\pi^{*} \cap$ kernel $\left.f^{*}\right)$, ([1], p. 429). Let $f^{\prime}: B U(n) \rightarrow K\left(\mathbf{Z}, 2(k-p+1) ; \mathbf{Z}, 2 k, \delta P^{1}\right)$ be a lifting of $c(k-p+1)$, $f_{k-p+1}: W_{n, q} \rightarrow K\left(Z, 2 k-2 p+1 ; \mathbf{Z}, 2 k-1, \delta P^{1}\right)$ a lifting of $a_{k-p+1}$, and set $\delta P_{\omega}^{1}(c(k-p+1))=\delta P_{f^{\prime} f}^{1}(\iota)$. If $[g]$ in $\pi_{2 k-1}\left(W_{n, q}\right)$ is a generator, then $f_{k-p+1 \#}[g] \in \delta P_{g}^{1}\left(a_{k-p+1}\right)$, [3]. Since $P^{1} a_{k-p+1}=\mu_{p}(k) a_{k}$, where $\mu_{p}(k) \equiv$ $k(\bmod p),([1]$, p. 429$)$, a direct computation of the operation $\delta P_{g}^{1}\left(a_{k-p+1}\right)$ yields the equation $f_{k-p+1 *}[g] \equiv \mu_{p}(k) p^{-1} m_{k}\left(\bmod m_{k}\right)$. If $c_{k}(\omega)=0$, then $m_{k} O^{2 k}(f)=0$ and the action of the homomorphisms $f_{k-p+1 *}$ on the obstruction is independent of the lifting. The next proposition follows immediately from the above remarks and Proposition 2.2. The assumption $p \leqq q$ forces the inequality of the proposition because we have $2 q \leqq n$ in our theorems.

Proposition 3.1. Let $p$ be a prime such that $p \leqq q$. If $c_{k}(\omega)=0$ and $m_{k} \equiv 0(\bmod p)$, then

$$
\mu_{p}(k) p^{-1} m_{k} O^{2 k}(f) \equiv \delta P_{\omega}^{1}(c(k-p+1)) \equiv \delta P_{f}^{1}(c(k-p+1)) .
$$

We now turn to the proof of Theorem 2. The proof of Theorem 1 will be discussed later. In addition to the properties of $W_{n, 4}$ mentioned above, we will need the fact that the 3-component of $\pi_{2 n-4}\left(W_{n, 4}\right)$ is zero if $n \neq 1(\bmod 3)[2]$ and some more precise information on the image of the Hurewicz homomorphism $h: \pi_{2 n-3}\left(W_{n, 4}\right) \rightarrow H_{2 n-3}\left(W_{n, 4} ; \mathbf{Z}\right), n$ odd and $n \geqq 9: m_{n-1} \equiv 0(\bmod 3)$ and $m_{n-1} \not \equiv 0(\bmod 9)$ if $n \not \equiv 1(\bmod 3)$, [4].

The conditions in Theorem 2 are clearly necessary. The hypothesis $c_{n-3}(\omega)=0$ implies that $O^{2 n-4}(f)$ is nonvoid and because $n$ is odd and $m_{n-2}=2$, Proposition 3.1 implies that $O^{2 n-4}(f)=\delta S q_{\omega}^{2}(c(n-3))$. This equation is an actual equality because the indeterminacy of $O^{2 n-4}(f)$ is image $\delta S q^{2}\left([10]\right.$, p. 191) which is the indeterminacy of $\delta S q_{\omega}^{2}(c(n-3))$. Therefore the hypothesis $0 \in \delta S q_{\omega}^{2}(c(n-3))$ is enough to imply that $O^{2 n-2}(f)$ is nonvoid since $n \neq 1(\bmod 3)$ means that $\pi_{2 n-4}\left(W_{n, 4}\right)$ has no 
3-component and $M$ is 3-connected $\bmod 2$. Since $c_{n-1}(\omega)=0$ and $m_{n-1} \equiv 0(\bmod 3), \quad$ we have $\mu_{3}(n-1) 3^{-1} m_{n-1} O^{2 n-2}(f) \equiv \delta P_{\omega}^{1}(c(n-3))$, where $\mu_{3}(n-1) \neq 0$ because $n \neq 1(\bmod 3)$. The proof of Theorem 2 will be complete when we have shown that this equation is an actual equality, because the other obstructions vanish by connectivity, Poincaré duality, and $c_{n}(\omega)=0$.

To establish equality, we work with the equation in the form $f_{n-3 *} O^{2 n-2}(f) \equiv O^{2 n-2}\left(f^{\prime} f\right), \quad$ where $\quad f_{n-3}: W_{n, 4} \rightarrow K(\mathbf{Z}, 2 n-7 ; \mathbf{Z}, 2 n-$ $\left.3, \delta P^{1}\right)$ and $f^{\prime}: B U(n) \rightarrow K\left(\mathbf{Z}, 2 n-6 ; \mathbf{Z}, 2 n-2, \delta P^{1}\right)$ are liftings of $a_{n-3}$ and $c(n-3)$, respectively. Let $h$ and $\bar{h}$ be $2 n-3$-liftings of $f^{\prime} f$ and $f$, respectively, and let $\left\{c^{2 n-2}(h)\right\}$ and $\left\{c^{2 n-2}(\bar{h})\right\}$ be the obstruction cohomology classes determined by these liftings. We assert that $h$ and $\bar{h}$ can be altered in such a way that the obstruction class of $h$ is unchanged and $f_{n-3 *}\left\{c^{2 n-2}(\bar{h})\right\}=\left\{c^{2 n-2}(h)\right\}$. The argument begins by altering $h$ by a $2 n-7$-cocycle $\nu$ such that $\{\nu\}=-3 O^{2 n-7}\left(f^{\prime} \bar{h}, h\right)$. The altered map $h_{\nu}$ extends to a $2 n-3$ lifting of $f^{\prime} f$ because of the homotopy of $K(\mathbf{Z}, 2 n-$ $\left.7 ; \mathbf{Z}, 2 n-3, \delta P^{1}\right)$ and $\left\{c^{2 n-2}(h)\right\}-\left\{c^{2 n-2}\left(h_{\nu}\right)\right\}=\delta P^{1} O^{2 n-7}\left(h, h_{\nu}\right)=\delta P^{1}\{\nu\}$, [9], so $\left\{c^{2 n-2}(h)\right\}=\left\{c^{2 n-2}\left(h_{\nu}\right)\right\}$ by our choice of $\nu$. Note that

$$
O^{2 n-7}\left(f^{\prime} \bar{h}, h_{\nu}\right)=O^{2 n-7}\left(f^{\prime} \bar{h}, h\right)+O^{2 n-7}\left(h, h_{\nu}\right)=-2 O^{2 n-7}\left(f^{\prime} \bar{h}, h\right) .
$$

The homomorphism $f_{n-3 \#}: \pi_{2 n-7}\left(W_{n, 4}\right) \rightarrow \mathbf{Z}$ is the identity and so we may alter $\bar{h}$ by a $2 n-7$-cocycle $\alpha$ such that $f_{n-3 *}\{\alpha\}=\{\alpha\}=O^{2 n-7}\left(f^{\prime} \bar{h}, h_{\nu}\right)$. If $\bar{h}_{\alpha}$ is the altered map, $O^{2 n-7}\left(\bar{h}, \bar{h}_{\alpha}\right)=-2 O^{2 n-7}\left(f^{\prime} \bar{h}, h\right)$, and so $\left\{c^{2 n-4}(\bar{h})\right\}-\left\{c^{2 n-4}\left(\bar{h}_{\alpha}\right)\right\}=\delta S q^{2} O^{2 n-7}\left(\bar{h}, \bar{h}_{\alpha}\right)=0$. Therefore, $\bar{h}_{\alpha}$ extends to a $2 n-3$ lifting of $f$ because $\left\{c^{2 n-4}(\bar{h})\right\}=0$ and $M$ is 3 -connected mod 2. We have

$$
O^{2 n-7}\left(f^{\prime} \bar{h}_{\alpha}, h_{\nu}\right)=O^{2 n-7}\left(f^{\prime} \bar{h}_{\alpha}, f^{\prime} \bar{h}\right)+O^{2 n-7}\left(f^{\prime} \bar{h}, h_{\nu}\right)=0
$$

because $O^{2 n-7}\left(\bar{h}_{\alpha}, \bar{h}\right)=-\{\alpha\}$, and so $f^{\prime} \bar{h}_{\alpha}$ is homotopic to $h_{\nu}$ in dimensions less than $2 n-3$ and the difference formula for cocycles yields $f_{n-3 *}\left\{c^{2 n-2}\left(\bar{h}_{\alpha}\right)\right\}=\left\{c^{2 n-2}\left(h_{\nu}\right)\right\}$. This completes the proof of equality and of Theorem 2. The proof of Theorem 1 is exactly the same except that there is no obstruction of order 3 . The remark about the special case $n \neq 0(\bmod 3), n \equiv 2(\bmod 4)$ follows from [2].

The problem of computing the operations $\delta P_{\omega}^{1}$ seems difficult. The following example shows that they are nontrivial invariants of the sectioning problem on the level of complexes ${ }^{1}$. Let $n$ be even, $n \geqq 6$, and let $p: E \rightarrow B U(n)$ be the first stage in the Postnikov system for the fibration $B U(n-3) \rightarrow B U(n)$ and let $p_{1}: E_{1} \rightarrow B U(n-2)$ be the first

${ }^{1}$ I am grateful to the referee for this example. 
stage in the system of the fibration $B U(n-3) \rightarrow B U(n-2)$. Let $X$ be the $2 n-1$-skeleton of $E_{1}$ and $\omega$ the bundle classified by the inclusion $B U(n-2) \rightarrow B U(n)$ composed with $p_{1}$. If $k$ in $H^{2 n-2}(E ; \mathbf{Z})$ is the $k$-invariant, then $2 k=p^{*} c(n-1)[10]$ and if $s: E_{1} \rightarrow E$ is the natural map, then $s^{*} k$ is in the image of the Bockstein mod 2. With these observations, it is easy to see that $\omega$ has the following properties: $c_{i}(\omega)=0, n-2 \leqq i \leqq n$, complex span $\omega \geqq 2$, but $0 \notin \delta S q_{\omega}^{2}(c(n-2))$ because $X$ does not lift to $B U(n-3)$.

\section{REFERENCES}

1. A. Borel and J.-P. Serre, Groupes de Lie et puissances réduites de Steenrod, Amer. J. Math., 75 (1953), 409-448.

2: M. Gilmore, Complex Stiefel manifolds, some homotopy groups and vector fields, Bull. Amer. Math. Soc., 73 (1967), 630-633.

3. R. D. Little, A relation between obstructions and functional cohomology operations, Proc. Amer. Math. Soc., 49 (1975), 259-264.

4. R. D. Little, Obstruction formulas and almost-complex manifolds, Proc. Amer. Math. Soc., 50 (1975), 459-462.

5. J.-P. Meyer, Functional cohomology operations and relations, Amer. J. Math., 97 (1965), 649-683.

6. R. E. Mosher and M. C. Tangora, Cohomology Operations and Applications in Homotopy Theory, Harper and Row, 1968.

7. P. Olum, Invariants for effective homotopy classification and extension of mappings, Mem. Amer. Math. Soc., 37 (1961).

8. - Factorizations and induced homomorphisms, Advances in Math., 3 (1969), 72-100.

9. - Seminar in obstruction theory, Cornell Univ. Notes, 1968.

10. E. Thomas, Postnikov invariants and higher order cohomology operations, Ann. of Math., 85 (1967), 184-217.

11. Real and complex vector fields on manifolds, J. Math. and Mech., 16 (1967), 1183-1206.

Received June 12, 1974.

UNIVERSITY OF HAWAII-MANOA 


\section{PACIFIC JOURNAL OF MATHEMATICS}

\section{EDITORS}

RICHARD ARENS (Managing Editor)

University of California

Los Angeles, California 90024

\section{J. Dugundu}

Department of Mathematics University of Southern California Los Angeles, California 90007

D. Gilbarg and J. Milgram Stanford University Stanford, California 94305

\section{ASSOCIATE EDITORS}
E. F. BECKENBACH
B. H. NeumanN
F. WOLF
K. YoSHIDA

\section{SUPPORTING INSTITUTIONS}

UNIVERSITY OF BRITISH COLUMBIA CALIFORNIA INSTITUTE OF TECHNOLOGY

UNIVERSITY OF CALIFORNIA

MONTANA STATE UNIVERSITY

UNIVERSITY OF NEVADA

NEW MEXICO STATE UNIVERSITY

OREGON STATE UNIVERSITY

UNIVERSITY OF OREGON

OSAKA UNIVERSITY

\author{
UNIVERSITY OF SOUTHERN CALIFORNIA \\ STANFORD UNIVERSITY \\ UNIVERSITY OF HAWAII \\ UNIVERSITY OF TOKYO \\ UNIVERSITY OF UTAH \\ WASHINGTON STATE UNIVERSITY \\ UNIVERSITY OF WASHINGTON \\ AMERICAN MATHEMATICAL SOCIETY
}

The Supporting Institutions listed above contribute to the cost of publication of this Journal, but they are not owners or publishers and have no responsibility for its contents or policies.

Mathematical papers intended for publication in the Pacific Journal of Mathematics should be in typed form or offset-reproduced (not dittoed), double spaced with large margins. Underline Greek letters in red, German in green, and script in blue. The first paragraph or two must be capable of being used separately as a synopsis of the entire paper. Items of the bibliography should not be cited there unless absolutely necessary, in which case they must be identified by author and Journal, rather than by item number. Manuscripts, in duplicate, may be sent to any one of the four editors. Please classify according to the scheme of Math. Reviews, Index to Vol. 39. All other communications should be addressed to the managing editor, or Elaine Barth, University of California, Los Angeles, California, 90024.

100 reprints are provided free for each article, only if page charges have been substantially paid. Additional copies may be obtained at cost in multiples of 50 .

The Pacific Journal of Mathematics is issued monthly as of January 1966. Regular subscription rate: $\$ 72.00$ a year (6 Vols., 12 issues). Special rate: $\$ 36.00$ a year to individual members of supporting institutions.

Subscriptions, orders for back numbers, and changes of address should be sent to Pacific Journal of Mathematics, 103 Highland Boulevard, Berkeley, California, 94708.

PUBLISHED BY PACIFIC JOURNAL OF MATHEMATICS, A NON-PROFIT CORPORATION

Printed at Jerusalem Academic Press, POB 2390, Jerusalem, Israel.

$$
\begin{gathered}
\text { Copyright } 1976 \text { Pacific Journal of Mathematics } \\
\text { All Rights Reserved }
\end{gathered}
$$




\section{Pacific Journal of Mathematics}

Vol. 62, No. 2

February, 1976

Allan Russell Adler and Catarina Isabel Kiefe, Pseudofinite fields, procyclic

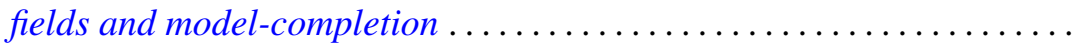

Christopher Allday, The stratification of compact connected Lie group

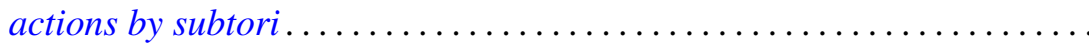

Martin Bartelt, Commutants of multipliers and translation operators .......

Herbert Stanley Bear, Jr., Ordered Gleason parts ..................

James Robert Boone, On irreducible spaces. II .....................

James Robert Boone, On the cardinality relationships between discrete

collections and open covers ............................

L. S. Dube, On finite Hankel transformation of generalized functions .......

Michael Freedman, Uniqueness theorems for taut submanifolds . . . . . . . . .

Shmuel Friedland and Raphael Loewy, Subspaces of symmetric matrices

containing matrices with a multiple first eigenvalue .............

Theodore William Gamelin, Uniform algebras spanned by Hartogs

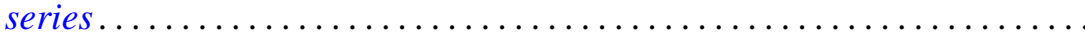

James Guyker, On partial isometries with no isometric part ............

Shigeru Hasegawa and Ryōtarō Satō, A general ratio ergodic theorem for

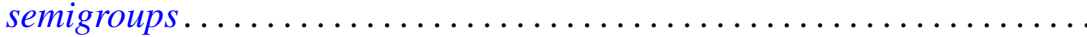

Nigel Kalton and G. V. Wood, Homomorphisms of group algebras with norm less than $\sqrt{2}$.

Thomas Laffey, On the structure of algebraic algebras...

Will Y. K. Lee, On a correctness class of the Bessel type differential operator $S_{\mu}$

Robert D. Little, Complex vector fields and divisible Chern classes ....

Kenneth Louden, Maximal quotient rings of ring extensions . .

Dieter Lutz, Scalar spectral operators, ordered $l^{\rho}$-direct sums, and the

counterexample of Kakutani-McCarthy . .

Ralph Tyrrell Rockafellar and Roger Jean-Baptiste Robert Wets, Stochastic

convex programming: singular multipliers and extended duality

singular multipliers and duality.

Edward Barry Saff and Richard Steven Varga, Geometric overconvergence of rational functions in unbounded domains ..........

Joel Linn Schiff, Isomorphisms between harmonic and P-harmonic Hardy

spaces on Riemann surfaces.

Virinda Mohan Sehgal and S. P. Singh, On a fixed point theorem of

Krasnoselskii for locally convex spaces.

Lewis Shilane, Filtered spaces admitting spectral sequence operations

Michel Smith, Generating large indecomposable continua . 